\title{
DIAMETER PRESERVING MAPS ON FUNCTION SPACES
}

\author{
MALIHEH HOSSEINI AND JUAN J. FONT
}

\begin{abstract}
In this paper we describe, under certain assumptions, surjective diameter preserving mappings when defined between function spaces, not necessarily algebras, thus extending most of the previous results for these operators. We provide an example which shows that our assumptions are not redundant.
\end{abstract}

\section{Introduction And Preliminaries}

In 1998, Győry and Molnár ([16]) introduced a new kind of linear operators based on the preservation of the diameter of the range of the functions. Since then, several papers have been published extending the scope of application of diameter preserving mappings (see e.g., [15], [8], [9] [18], [7], [13], [2], [1], [3], and [4]).

Diameter preserving mappings are indeed isometries if we consider the underlying spaces of functions endowed with the diameter (semi) norm. So, it is apparent that the description of the extreme points of the closed unit ball of the dual of the spaces endowed with the diameter norm is the key to study such maps. González and Uspenskij ([15]) and, independently, Cabello ([8]) characterized the extreme points of the closed unit ball of the dual of $C(X)$ ( $X$ compact) endowed with the diameter norm. However, if we consider linear subspaces of $C(X)$ (function spaces), the situation might be more intricate. Namely, in [14] (see also [6]), the authors describe such extreme points when the function spaces satisfy the Unique Decomposition property, although several examples confirm that a complete characterization seems to be complicated.

In this paper, we first analyse the diametral boundary of a function space, a concept which was just proposed (without any study) in [3] and which is based on the extreme points cited above. We check that it is indeed a boundary and show that it is a useful tool for the description of the diameter preserving surjections in this context. We also provide a characterization of the extreme points for several types of subspaces, which include extremely regular function spaces, Thanks to these results, we can describe diameter preserving surjections defined between such function spaces, thus extending most of the previous results for these maps.

2010 Mathematics Subject Classification. Primary 47B38; Secondary 46J10, 47B33.

Key words and phrases: Diameter-preserving maps, function spaces, Choquet boundary.

Research of J.J. Font was partially supported by the Spanish Ministry of Science and Education (Grant number MTM2011-23118), and by Bancaixa (Projecte P11B2011-30). 
We first need to state some preliminary definitions. Let $X$ be a compact (Hausdorff) space and let $C(X)$ be the algebra of all scalar-valued continuous functions on $X$ endowed with the supremum norm $\|\cdot\|_{\infty}$.

A linear subspace $A$ of $C(X)$ is called a function space on $X$ if $A$ separates the points of $X$ and contains the set of constant functions, denoted by $C$. A function space $A$ on $X$ is called a function algebra if it is an algebra. $\bar{A}$ will denote the closure of $A$ in $\left(C(X),\|\cdot\|_{\infty}\right)$.

Given a linear subspace $A$ of $C(X)$, the Choquet boundary, $C h(A)$, of $A$ is the set of all $x \in X$ for which $\delta_{x}$, the evaluation functional at $x$, is an extreme point of the unit ball of the dual space of $\left(A,\|\cdot\|_{\infty}\right)$. Clearly, $C h(A)=C h(\bar{A})$. If $x, x^{\prime} \in C h(A)$, then we shall write $\delta_{x, x^{\prime}}:=\delta_{x}-\delta_{x^{\prime}}$.

For any function $f \in C(X), \operatorname{diam}(f)$ denotes the diameter of the range of $f$. For two function spaces $A$ and $B$, a linear map $T: A \rightarrow B$ is called diameter preserving if $\operatorname{diam}(f)=\operatorname{diam}(T f)$ for all $f \in A$.

For a function space $A$ on a compact space $X, A_{d}$ stands for the quotient space $A / C$ endowed with the diameter norm, $\|\pi(f)\|_{d}:=\operatorname{diam}(f)$ for all $f \in A$, where $\pi$ is the quotient map $\pi: A \rightarrow A / C$, and $\left(A_{d}^{*},\|\cdot\|_{d}^{*}\right)$ its dual space. Moreover, we denote the unit ball of the dual space $A_{d}^{*}$ by $\mathcal{B}_{A_{d}^{*}}$ and the set of its extreme points by $\operatorname{ext}\left(\mathcal{B}_{A_{d}^{*}}\right)$. Since $A_{d}$ is isomorphic to a quotient of $A$, then $A_{d}^{*}$ is isomorphic to a subspace of $A^{*}=\left(A,\|\cdot\|_{\infty}\right)^{*}$. In fact, $A_{d}^{*}=\left\{\mu \in A^{*}: \mu(X)=0\right\}$.

Let $A$ be a function space on $X$ and $x \in X$. A representing measure for $\delta_{x}$ is a positive measure $\mu$ on $X$ such that $\delta_{x}(f)=\int_{X} f d \mu$ for all $f \in A$. We use the same notation, $\delta_{x}$, for the evaluation functional at $x$ and the unit point mass at $x$.

\section{EXTREME POINTS}

Let us suppose, in this section, that $A$ is a function space on a compact space $X$. Notice that by arguments similar to those in the proof of [14, Theorem 1], we can regard $\operatorname{ext}\left(\mathcal{B}_{A_{d}^{*}}\right)$ as a nonempty subset of $\left\{\alpha \delta_{x, x^{\prime}}: x, x^{\prime} \in C h(A), x \neq x^{\prime}, \alpha \in \mathbb{T}\right\}$ and that this inclusion may be proper. As a consequence, the following set, which was proposed in [3] without any study, can be defined:

Definition 2.1. The diametral boundary of $A$ is defined as follows:

$$
d \operatorname{ch}(A):=\left\{\left\{x_{1}, x_{2}\right\}: \delta_{x_{1}, x_{2}} \in \operatorname{ext}\left(\mathcal{B}_{A_{d}^{*}}\right)\right\}
$$

On the other hand, we can define the following linear subspace of $C(X \times X)$ :

$$
A-A:=\left\{h \in C(X \times X): h(x, y)=h_{1}(x)-h_{1}(y), h_{1} \in A\right\}
$$

endowed with the supremum norm.

Proposition 2.2. $\left\{x_{1}, x_{2}\right\} \in d \operatorname{ch}(A)$ if and only if $\left(x_{1}, x_{2}\right) \in C h(A-A)$. 
Proof. Consider the linear operator $L: A_{d} \longrightarrow(A-A)$ defined as

$$
L(\pi(f))(x, y):=f(x)-f(y) .
$$

Since $\|\pi(f)\|_{d}=\|L(f)\|_{\infty}$, we have that $L$ is a linear onto isometry. As a consequence, the adjoint map $L^{*}:(A-A)^{*} \longrightarrow A_{d}^{*}$ sends the extreme points of the closed unit ball of $(A-A)^{*}$ onto the extreme points of the closed unit ball of $A_{d}^{*}$. That is, the extreme points of $A_{d}^{*}$ can be identified with $C h(A-A)$.

It is well known the Choquet boundary of each linear subspace $B$ of continuous functions is a boundary for $B$ in the sense that every element of $B$ attains its maximum value on it. Hence, from Proposition 2.2, we can deduce that $d c h(A)$ is a boundary for $\left(A_{d},\|\cdot\|_{d}\right)$ in the sense that, given $f \in A$, then $\|\pi(f)\|_{d}=\left|f\left(x_{1}\right)-f\left(x_{2}\right)\right|$ for some $\left\{x_{1}, x_{2}\right\} \in d c h(A)$.

Let us now describe the diametral boundary of several function spaces:

- ([15], [8]) Let $A=C(X)$. Then $d \operatorname{ch}(C(X))=\left\{\left\{x_{1}, x_{2}\right\}: x_{1}, x_{2} \in X\right\}$.

- ([14]) If A satifies the Unique Decomposition property, then $d c h(A)=\left\{\left\{x_{1}, x_{2}\right\}: x_{1}, x_{2} \in\right.$ $C h(A)\}$. Indeed, this is a characterization of the Unique Decomposition property $([6])$.

- ([12]) If $A$ is a function space on a compact space $X$ which is dense in $\left(C(X),\|\cdot\|_{\infty}\right)$, then $d c h(A)=\left\{\left\{x_{1}, x_{2}\right\}: x_{1}, x_{2} \in X\right\}$.

- ([13]) If $A$ is a (real-valued) extremely regular function space on a compact space $X$ (see definition before Corollary 3.3), then $\operatorname{dch}(A)=\left\{\left\{x_{1}, x_{2}\right\}: x_{1}, x_{2} \in X\right\}$.

However, the description of the diametral boundary of a function space is not always so clear as the following example shows. First, we need a technical result which we shall also use in the proof of our main result:

Lemma 2.3. Let $u \in C(X)$ with $0 \leq u \leq 1$. Let $x_{0} \in X$ and $U$ a neighbourhood of $x_{0}$ such that $u\left(x_{0}\right)=1$ and $u(X \backslash U)=0$. If $x_{0} \in C h(A)$ for a certain function space $A$ on $X$, then

$$
\sup \left\{\operatorname{Reh}\left(x_{0}\right): h \in \bar{A}, \operatorname{Re} h \leq u\right\}=1 .
$$

Proof. Let $r:=\sup \left\{\operatorname{Re} h\left(x_{0}\right): h \in \bar{A}, \operatorname{Re} h \leq u\right\}$. It is apparent that $0 \leq r \leq 1$. Now if $r \neq 1$, then $r=1-\epsilon$ for some $0<\epsilon \leq 1$. Then $u \notin \operatorname{Re} \bar{A}$ since $u\left(x_{0}\right)=1$. Consider the subspace $E$ of $C_{\mathbb{R}}(X)$ spanned by $\operatorname{Re} \bar{A}$ and $u$. Each element of $E$ can be written as $k+\lambda u$ for a unique $k \in \operatorname{Re} \bar{A}$ and $\lambda \in \mathbb{R}$. Define a linear functional $L$ on $E$ by $L(k+\lambda u):=k\left(x_{0}\right)+\lambda(1-\epsilon)$. Clearly, $L$ is a positive functional with $L(1)=1=\|L\|$. So, there exists a positive measure $\mu \in M(X)$ of total mass 1 such that $L(k+\lambda u)=\int(k+\lambda u) d \mu$ for all $k \in \operatorname{Re} \bar{A}$ and $\lambda \in \mathbb{R}$. Thus

$$
k\left(x_{0}\right)+\lambda(1-\epsilon)=\int k d \mu+\lambda \int_{3} u d \mu(k \in \operatorname{Re} \bar{A}, \lambda \in \mathbb{R})(*) .
$$


In particular, $k\left(x_{0}\right)=\int k d \mu$ for all $k \in \operatorname{Re} \bar{A}$ and, consequently, $f\left(x_{0}\right)=\int f d \mu$ for all $f \in \bar{A}$. This means that $\mu$ is a representing measure for $\delta_{x_{0}}$ and since $x_{0} \in C h(A)$, then, by [17, Corollary, P. 52], $\mu$ is the point mass at $x_{0}$. Therefore, by $(*), 1-\epsilon=\int u d \mu=u\left(x_{0}\right)$ and so $u\left(x_{0}\right)<1$, a contradiction showing that $r=1$.

Example 2.4. Let us consider the hexagon $S$ in the plane with vertices at the points $x_{1}=(1,0)$, $x_{2}=\left(\frac{1}{2}, \frac{1}{2}\right), x_{3}=\left(\frac{-1}{2}, \frac{1}{2}\right), x_{4}=(-1,0)$ and $x_{5}=\left(\frac{-1}{2}, \frac{-1}{2}\right), x_{6}=\left(\frac{1}{2}, \frac{-1}{2}\right)$. Let $A(S)$ be the space of affine continuous functions on the compact convex set $S$, that is, the linear subspace of $C(S)$ consisting of the functions of the form $f(x, y)=a x+b y+c(a, b, c \in \mathbb{C})$.

We shall first show that $C h(A(S))=\left\{x_{1}, x_{2}, x_{3}, x_{4}, x_{5}, x_{6}\right\}$. It is obvious that $C h(A(S)) \supseteq$ $\left\{x_{1}, x_{2}, x_{3}, x_{4}, x_{5}, x_{6}\right\}$. In order to check the opposite inclusion, suppose $z_{0}=\left(x_{0}, \frac{-1}{2}\right)$ is an arbitrary intermediate point on the edge $x_{5} x_{6}$ (vertices are $x_{5}$ and $x_{6}$ ). Since $\frac{-1}{2}<x_{0}<\frac{1}{2}$ we can choose a neighborhood $U$ of $z_{0}$ such that $\left(x_{0} \pm \epsilon, \frac{-1}{2}\right) \notin U$ for some $\epsilon>0$ with $\frac{-1}{2}<x_{0} \pm \epsilon<\frac{1}{2}$.

Let us check that $z_{0} \notin C h(A(S))$. Otherwise, take $u \in C(S)$ with $0 \leq u \leq 1$ with $u\left(z_{0}\right)=1$ and $u(S \backslash U)=0$. If $z_{0} \in C h(A(S))$, then, by Lemma 2.3, $\sup \left\{\operatorname{Re} f\left(z_{0}\right): f \in A(S), \operatorname{Re} f \leq u\right\}=1$. Thus there is a function $f \in A(S)$ with $\operatorname{Re} f\left(z_{0}\right)>\frac{3}{4}$ and $\operatorname{Re} f \leq u$. There are complex scalars $a, b, c$ such that $f(x, y)=a x+b y+c$ for all $(x, y) \in S$. In particular, $\operatorname{Re} f\left(x_{0} \pm \epsilon, \frac{-1}{2}\right) \leq 0$. Then $\operatorname{Re}\left(a\left(x_{0}+\epsilon\right)-\frac{b}{2}+c\right) \leq 0$ and $\operatorname{Re}\left(a\left(x_{0}-\epsilon\right)-\frac{b}{2}+c\right) \leq 0$, consequently $\operatorname{Re}\left(2 a x_{0}-b+2 c\right) \leq 0$, which is a contradiction with $\operatorname{Re} f\left(z_{0}\right)=\operatorname{Re}\left(a x_{0}-\frac{b}{2}+c\right)>\frac{3}{4}$. Hence $z_{0} \notin C h(A(S))$. Analogously, the intermediate points on the edge $x_{2} x_{3}$ do not belong to $C h(A(S))$. Now let $z_{0}=\left(x_{0}, y_{0}\right)$ be an intermediate point on the edge $x_{1} x_{6}$ or $x_{3} x_{4}$ (resp. $x_{1} x_{2}$ or $x_{4} x_{5}$ ) belonging to $C h(A(S)$ ). Take a neighborhood $U$ of $z_{0}$ such that $\left(x_{0} \pm \epsilon, y_{0} \pm \epsilon\right) \in S \backslash U$ (resp. $\left.\left(x_{0} \pm \epsilon, y_{0} \mp \epsilon\right) \in S \backslash U\right)$ for some $\epsilon>0$ and then, by a similar argument to the above, we obtain a contradiction. Therefore, $C h(A(S))=\left\{x_{1}, x_{2}, x_{3}, x_{4}, x_{5}, x_{6}\right\}$.

Since $\delta_{x_{4}, x_{5}}=\delta_{x_{2}, x_{1}}=\left(\delta_{x_{4}, x_{6}}+\delta_{x_{1}, x_{6}}\right) / 2, \delta_{x_{1}, x_{6}}=\delta_{x_{3}, x_{4}}=\left(\delta_{x_{1}, x_{5}}+\delta_{x_{4}, x_{5}}\right) / 2, \delta_{x_{2}, x_{3}}=\delta_{x_{6}, x_{5}}=$ $\left(\delta_{x_{2}, x_{4}}+\delta_{x_{5}, x_{4}}\right) / 2, \delta_{x_{3}, x_{5}}=\delta_{x_{2}, x_{6}}=\left(\delta_{x_{2}, x_{5}}+\delta_{x_{3}, x_{6}}\right) / 2, \delta_{x_{1}, x_{5}}=\delta_{x_{2}, x_{4}}=\left(\delta_{x_{1}, x_{4}}+\delta_{x_{2}, x_{5}}\right) / 2, \delta_{x_{4}, x_{6}}=$ $\delta_{x_{3}, x_{1}}=\left(\delta_{x_{4}, x_{1}}+\delta_{x_{3}, x_{6}}\right) / 2$, then $d \operatorname{ch}(A(S)) \subseteq\left\{\left\{x_{1}, x_{4}\right\},\left\{x_{2}, x_{5}\right\},\left\{x_{3}, x_{6}\right\}\right\}$.

Indeed, we claim that $\operatorname{ext}\left(\mathcal{B}_{A(S)_{d}^{*}}\right)=\left\{\alpha \delta_{x_{1}, x_{4}}, \alpha \delta_{x_{2}, x_{5}}, \alpha \delta_{x_{3}, x_{6}}: \alpha \in \mathbb{T}\right\}$. In order to see this, suppose that $\delta_{x_{1}, x_{4}} \notin \operatorname{ext}\left(\mathcal{B}_{A(S)_{d}^{*}}\right)$. Since $A(S)_{d}^{*}$ is a finite dimensional space, then $\mathcal{B}_{A(S)_{d}^{*}}=$ $\operatorname{co}\left(\operatorname{ext}\left(\mathcal{B}_{A(S)_{d}^{*}}\right)\right)$, and so we have

$$
\delta_{x_{1}, x_{4}}=t_{1} \beta_{1} \delta_{x_{2}, x_{5}}+t_{2} \beta_{2} \delta_{x_{2}, x_{5}}
$$

such that $\beta_{1}, \beta_{2} \in \mathbb{T}, t_{1}, t_{2} \in[0,1]$ and $t_{1}+t_{2}=1$. Thus for each $f(x, y)=a x+b y+c$,

$$
2 a=a\left(t_{1} \beta_{1}-t_{2} \beta_{2}\right)+b\left(t_{1} \beta_{1}+t_{2} \beta_{2}\right),
$$


which easily gives a contradiction. Similarly, $\delta_{x_{2}, x_{5}}, \delta_{x_{3}, x_{6}} \in \operatorname{ext}\left(\mathcal{B}_{A(S)_{d}^{*}}\right)$. Therefore,

$$
d \operatorname{ch}(A(S))=\left\{\left\{x_{1}, x_{4}\right\},\left\{x_{2}, x_{5}\right\},\left\{x_{3}, x_{6}\right\}\right\} \neq\left\{\left\{x, x^{\prime}\right\}: x, x^{\prime} \in C h(A(S))\right\} .
$$

\section{DiAMETER PRESERVING MAPPINGS}

In the sequel, given a function space $A$ on a compact space $X$, we set

$$
\widetilde{X}:=\left\{x \in C h(A):\left\{x, x^{\prime}\right\} \in d \operatorname{ch}(A) \text { for some } x^{\prime} \in C h(A)\right\} .
$$

As mentioned before, we can regard $\operatorname{ext}\left(\mathcal{B}_{A_{d}^{*}}\right)$ as a nonempty subset of $\left\{\alpha \delta_{x, x^{\prime}}: x, x^{\prime} \in C h(A), x \neq\right.$ $\left.x^{\prime}, \alpha \in \mathbb{T}\right\}$, and consequently $\widetilde{X}$ is a nonempty subset of $C h(A)$.

Theorem 3.1. Let $A_{i}, i=1,2$, be (complex or real-valued) function spaces on compact spaces $X_{i}$ satisfying the following conditions:

(i) the set $\left\{\delta_{x}: x \in X_{i}\right\}$ is linearly independent in $\left(A_{i},\|\cdot\|_{\infty}\right)^{*}$,

(ii) $\operatorname{dch}\left(A_{1}\right)=\left\{\left\{x, x^{\prime}\right\}: x, x^{\prime} \in \widetilde{X_{1}}\right\}$.

Assume that $T: A_{1} \rightarrow A_{2}$ is a surjective diameter preserving map. Then there are a homeomorphism $\psi: \widetilde{X_{2}} \rightarrow \widetilde{X_{1}}$, a scalar $\lambda \in \mathbb{T}$ and a linear functional $L: A_{1} \rightarrow \mathbb{C}$ such that $T f(y)=$ $\lambda f(\psi(y))+L(f)$ for all $f \in A_{1}$ and $y \in \widetilde{X_{2}}$.

Proof. Since $T$ is a diameter preserving map then it induces a linear isometry $T_{d}: A_{1 d} \rightarrow A_{2 d}$ under the diameter norm defined by $T_{d}(\pi(f))=\pi(T f)$ for all $f \in A_{1}$. Hence the adjoint of $T$, $T_{d}^{*}: A_{2 d}^{*} \rightarrow A_{1 d}^{*}$, is a linear bijective isometry and, so, $T_{d}^{*}\left(\operatorname{ext}\left(\mathcal{B}_{A_{2 d}^{*}}\right)\right)=\operatorname{ext}\left(\mathcal{B}_{A_{1 d}^{*}}\right)$.

We can now define a map $\Phi: d \operatorname{ch}\left(A_{1}\right) \rightarrow d \operatorname{ch}\left(A_{2}\right)$ by $\Phi\left\{x_{1}, x_{2}\right\}:=\operatorname{supp}\left(T_{d}^{*^{-1}}\left(\delta_{x_{1}, x_{2}}\right)\right)$ and claim that $\Phi$ is injective. Indeed, if $\left\{x_{1}, x_{2}\right\}$ and $\left\{x_{3}, x_{4}\right\}$ are distinct elements of $d c h\left(A_{1}\right)$ such that $\Phi\left\{x_{1}, x_{2}\right\}=\Phi\left\{x_{3}, x_{4}\right\}$, then $\delta_{x_{1}}-\delta_{x_{2}}=\gamma\left(\delta_{x_{3}}-\delta_{x_{4}}\right)$ for some $\gamma \in \mathbb{T}$. This linear combination contradicts condition (i). Moreover, $\Phi$ is surjective since $T_{d}^{*}\left(\operatorname{ext}\left(\mathcal{B}_{A_{2 d}^{*}}\right)\right)=\operatorname{ext}\left(\mathcal{B}_{A_{1 d}^{*}}\right)$.

Fix $x \in \widetilde{X_{1}}$. We shall next show that, for each pair of different points $x_{1}, x_{2} \in C h\left(A_{1}\right)$ distinct from $x$ and such that $\left\{x_{1}, x\right\},\left\{x, x_{2}\right\} \in d c h\left(A_{1}\right)$, we have $\operatorname{card}\left(\Phi\left\{x_{1}, x\right\} \cap \Phi\left\{x_{2}, x\right\}\right)=1$. It is apparent, due to the injectivity of $\Phi$, that $\operatorname{card}\left(\Phi\left\{x_{1}, x\right\} \cap \Phi\left\{x_{2}, x\right\}\right) \neq 2$. Therefore let us suppose that $\operatorname{card}\left(\Phi\left\{x_{1}, x\right\} \cap \Phi\left\{x_{2}, x\right\}\right)=0$. As, by condition (ii), $T_{d}^{*^{-1}}\left(\delta_{x_{1}, x}\right)-T_{d}^{*^{-1}}\left(\delta_{x, x_{2}}\right)=T_{d}^{*^{-1}}\left(\delta_{x_{1}, x_{2}}\right) \in$ $\operatorname{ext}\left(\mathcal{B}_{A_{2 d}^{*}}\right)$, it follows that there exists a nonzero linear combination in $\left\{\delta_{y}: y \in C h\left(A_{2}\right)\right\}$ since, as mentioned above, $\operatorname{ext}\left(\mathcal{B}_{A_{2 d}^{*}}\right)$ is included in the set $\left\{\alpha \delta_{y, y^{\prime}}: y, y^{\prime} \in C h\left(A_{2}\right), y \neq y^{\prime}, \alpha \in \mathbb{T}\right\}$. This contradicts again condition (i), which yields $\operatorname{card}\left(\Phi\left\{x_{1}, x\right\} \cap \Phi\left\{x_{2}, x\right\}\right)=1$. Let $\varphi(x)$ be the unique point in this intersection.

We must now prove that $\varphi(x) \in \Phi\left\{x, x^{\prime}\right\}$ for each $x^{\prime} \in \widetilde{X_{1}}$. Contrary to what we claim, assume that there exists $x_{3} \in \widetilde{X_{1}} \backslash\left\{x, x_{1}, x_{2}\right\}$ such that $\varphi(x)$ does not belong to $\Phi\left\{x, x_{3}\right\}$. Reasoning as in 
the above paragraph and by condition (i), there exist distinct points $y_{1}, y_{2} \in C h\left(A_{2}\right) \backslash\{\varphi(x)\}$ with $\Phi\left\{x_{1}, x\right\}=\left\{\varphi(x), y_{1}\right\}, \Phi\left\{x_{2}, x\right\}=\left\{\varphi(x), y_{2}\right\}$ and $\Phi\left\{x_{3}, x\right\}=\left\{y_{1}, y_{2}\right\}$. Furthermore

$$
T_{d}^{*^{-1}}\left(\delta_{x_{1}}-\delta_{x_{2}}\right)=T_{d}^{*^{-1}}\left(\delta_{x_{1}, x}\right)-T_{d}^{*^{-1}}\left(\delta_{x_{2}, x}\right)=\beta \delta_{y_{1}, y_{2}}
$$

for some $\beta \in \mathbb{T}$. Then $\Phi\left\{x_{1}, x_{2}\right\}=\left\{y_{1}, y_{2}\right\}$, which contradicts the injectivity of $\Phi$.

Therefore, we may conclude that there exist $\beta \in \mathbb{T}$ and an injective map $\varphi: \widetilde{X_{1}} \rightarrow C h\left(A_{2}\right)$ such that for each $x, x^{\prime} \in \widetilde{X_{1}}, T_{d}^{*-1}\left(\delta_{x, x^{\prime}}\right)=\beta \delta_{\varphi(x), \varphi\left(x^{\prime}\right)}$.

It is not difficult to see $\varphi\left(\widetilde{X_{1}}\right)=\widetilde{X_{2}}$ since $A_{2}$ satisfies condition (i). Now let $\psi:=\varphi^{-1}: \widetilde{X_{2}} \rightarrow \widetilde{X_{1}}$. Then, $\psi$ is a bijective map such that for all $y, y^{\prime} \in \widetilde{X_{2}}, T_{d}^{*}\left(\delta_{y, y^{\prime}}\right)=\lambda \delta_{\psi(y), \psi\left(y^{\prime}\right)}$, where $\lambda=\bar{\beta}$. Hence, $T_{d}^{*}\left(\delta_{y}-\delta_{y^{\prime}}\right)=\lambda\left(\delta_{\psi(y)}-\delta_{\psi\left(y^{\prime}\right)}\right)$ and so for each $f \in A_{1}$ and $y, y^{\prime} \in \widetilde{X_{2}}, T f(y)-T f\left(y^{\prime}\right)=$ $\lambda\left(f(\psi(y))-f\left(\psi\left(y^{\prime}\right)\right)\right)$. Now, by fixing $y^{\prime} \in \widetilde{X_{2}}$, we can define a linear functional $L: A_{1} \rightarrow \mathbb{C}$ as $L(f):=-\lambda f\left(\psi\left(y^{\prime}\right)\right)+T f\left(y^{\prime}\right)$ for all $f \in A_{1}$. Thus, for all $f \in A_{1}$ and $y \in \widetilde{X_{2}}, T f(y)=$ $\lambda f(\psi(y))+L(f)$.

Finally we shall show that $\psi$ is a homeomorphism. To see the continuity of $\psi$, let $\left(y_{i}\right)_{i}$ be a convergent net in $\widetilde{X_{2}}$ to $y_{0}$ such that $\left(\psi\left(y_{i}\right)\right)_{i}$ converges to $x_{0}$ in $X_{1}$. It is enough to show that $x_{0}=\psi\left(y_{0}\right)$. Contrary to what we claim, let us suppose that there is a neighborhood $U$ of $\psi\left(y_{0}\right)$ such that $x_{0} \in X_{1} \backslash U$. Take $u \in C\left(X_{1}\right)$ with $0 \leq u \leq 1$ on $X_{1}, u\left(\psi\left(y_{0}\right)\right)=1$ and $u=0$ on $X_{1} \backslash U$. Since $\psi\left(y_{0}\right) \in C h\left(A_{1}\right)$, we know, by Lemma 2.3 , that $\sup \left\{\operatorname{Re} h\left(\psi\left(y_{0}\right)\right): h \in \overline{A_{1}}\right.$, Reh $\left.\leq u\right\}=1$. Hence, it is not difficult to see that $\sup \left\{\operatorname{Re} h\left(\psi\left(y_{0}\right)\right): h \in A_{1}, \operatorname{Re} h \leq u\right\}=1$. Then there exists $h \in A_{1}$ with $\operatorname{Re} h \leq u$ and $\operatorname{Re} h\left(\psi\left(y_{0}\right)\right)>\frac{3}{4}$. We can consider $i_{0}$ such that, for all $i \geq i_{0},\left|T h\left(y_{i}\right)-T h\left(y_{0}\right)\right|<\frac{1}{4}$. On the other hand, since $\lim _{i}\left|T h\left(y_{i}\right)-T h\left(y_{0}\right)\right|=\lim _{i}\left|h\left(\psi\left(y_{i}\right)\right)-h\left(\psi\left(y_{0}\right)\right)\right|=\left|h\left(x_{0}\right)-h\left(\psi\left(y_{0}\right)\right)\right| \geq$ $\frac{3}{4}-\operatorname{Re} h\left(x_{0}\right) \geq \frac{3}{4}$, then, for a sufficiently large index $i,\left|T h\left(y_{i}\right)-T h\left(y_{0}\right)\right|>\frac{1}{4}$, which is a contradiction. Thus $x_{0}=\psi\left(y_{0}\right)$ and, consequently, $\psi$ is continuous.

Similarly, we prove that $\varphi\left(\psi^{-1}\right)$ is continuous. Suppose, on the contrary, that $\left(x_{i}\right)_{i}$ is a convergent net in $\widetilde{X_{1}}$ to $x_{0}$ such that $\left(\varphi\left(x_{i}\right)\right)_{i}$ converges to $y_{0}$ in $X_{2}$ and $y_{0} \neq \varphi\left(x_{0}\right)$. As above, we can choose $k \in A_{2}$ with $\operatorname{Re} k\left(y_{0}\right) \leq 0$ and $\operatorname{Re} k\left(\varphi\left(x_{0}\right)\right)>\frac{3}{4}$. Then taking $h^{\prime} \in A_{1}$ such that $k=T h^{\prime}$, from

$$
\lim _{i}\left|h^{\prime}\left(x_{i}\right)-h^{\prime}\left(x_{0}\right)\right|=\lim _{i}\left|k\left(\varphi\left(x_{i}\right)\right)-k\left(\varphi\left(x_{0}\right)\right)\right|=\left|k\left(y_{0}\right)-k\left(\varphi\left(x_{0}\right)\right)\right| \geq \frac{3}{4}-\operatorname{Re} k\left(y_{0}\right) \geq \frac{3}{4},
$$

we get a contradiction. Therefore, $\psi$ is a homeomorphism.

Remark 3.2. (1) Condition (i) in Theorem 3.1 is true if, for instance, $A_{1}$ and $A_{2}$ are function algebras, although it is not true for general function spaces as Example 2.4 shows. Indeed, it is a routine matter to verify that $\delta_{x_{1}}=\delta_{x_{2}}-\delta_{x_{3}}$, which proves that $A(S)$ does not satisfy condition (i). 
Condition (ii) in Theorem 3.1 is true if, for instance, $\operatorname{ext}\left(\mathcal{B}_{A_{1 d}^{*}}\right)=\left\{\alpha \delta_{x, x^{\prime}}: x, x^{\prime} \in C h\left(A_{1}\right), x \neq\right.$ $\left.x^{\prime}, \alpha \in \mathbb{T}\right\}$, although it is not true for general function spaces as can be deduced from Example 2.4 too.

(2) The following example, adapted from [7, p. 134], shows that assumptions (i) and (ii) in our main result (Theorem 3.1) cannot be relaxed:

Let $A(K)$ be the space of affine continuous functions on the compact convex set

$$
K:=\operatorname{co}\{(1,0),(-1,0),(0,1)\}
$$

Similarly, let us also consider $A(S)$, where $S$ is the hexagon which appears in Example 2.4 and, thus, we already know that $A(S)$ satisfies neither condition (i) nor condition (ii) in Theorem 3.1 . Moreover, notice that $\widetilde{S}=\left\{x_{1}, x_{2}, x_{3}, x_{4}, x_{5}, x_{6}\right\}$. An argument similar to Example 2.4 (even easier) yields $C h(A(K))=\{(1,0),(-1,0),(0,1)\}, d c h(A(K))=\left\{\left\{x, x^{\prime}\right\}: x, x^{\prime} \in C h(A(K)), x \neq x^{\prime}\right\}$ and, consequently, $\widetilde{K}=C h(A(K))$. Especially, we see that $A(K)$ satisfies both condition (i) and condition (ii) in the theorem.

Then, according to [7, p. 134], there is a linear diameter preserving bijection between $A(K)$ and $A(S)$, while it is clear that there is no homeomorphism between between $\widetilde{K}$ and $\widetilde{S}$. As a consequence of the above paragraph, it is apparent, taking $A_{1}=A(K)$ and $A_{2}=A(S)$, that condition (i) in Theorem 3.1 is not redundant. Furthermore, condition (ii) cannot be removed either. Indeed, if we take $A_{1}=A(S)$ and $A_{2}=A(K)$, we note that condition (i) for $A_{1}$ in such theorem is used only to obtain the injectivity of $\Phi$ in the proof, but in this particular case we can check directly that $\Phi$ is injective (because $\operatorname{card}(d c h(A(S)))=\operatorname{card}(d c h(A(K)))=3$ and $T_{d}^{*}$ is a bijective isometry). Hence, since the result is not valid in this case, we conclude the necessity of condition (ii).

We are now showing that Theorem 3.1 can be applied to several types of function spaces.

It is said (see, e.g., [10]) that a function space $A$ on $X$ is extremely regular if for each $x \in X$, each neighborhood $V$ of $x$ and each scalar $\epsilon$ with $0<\epsilon<1$, there exists a function $f \in A$ with $\|f\|_{\infty}=f(x)=1$ and $|f|<\epsilon$ on $X \backslash V$.

Corollary 3.3. Let $A$ and $B$ be (real-valued) extremely regular function spaces on compact spaces $X$ and $Y$, respectively, and $T: A \rightarrow B$ be a surjective linear diameter preserving map, then there are a homeomorphism $\psi: Y \rightarrow X$, a scalar $\lambda \in\{ \pm 1\}$ and also a linear functional $L: A \rightarrow \mathbb{R}$ such that for all $f \in A, y \in Y, T f(y)=\lambda f(\psi(y))+L(f)$. 
Proof. Let $\alpha_{1}, \cdots, \alpha_{n} \in \mathbb{R} \backslash\{0\}$ and different points $x_{1}, \cdots, x_{n} \in X$ such that $\alpha_{1} \delta_{x_{1}}+\cdots+\alpha_{n} \delta_{x_{n}}=0$ on $A$. Let $V$ be a neighborhood of $x_{1}$ such that $x_{j} \notin V$ for all $j \in\{2, \cdots, n\}$. Choose $0<\beta<$ $\min \left(\left|\alpha_{1}\right|, 1\right)$. Then for $\epsilon=\frac{\beta}{\sum_{i=1}^{n}\left|\alpha_{i}\right|}$, there exists a function $f \in A$ with $f\left(x_{1}\right)=1=\|f\|_{\infty}$ and $|f|<\epsilon$ on $X \backslash V$. Consequently,

$$
0=\left|\alpha_{1} f\left(x_{1}\right)+\cdots+\alpha_{n} f\left(x_{n}\right)\right| \geq\left|\alpha_{1}\right|-\left|\alpha_{2} f\left(x_{2}\right)\right|-\cdots-\left|\alpha_{n} f\left(x_{n}\right)\right|>\left|\alpha_{1}\right|-\beta,
$$

and, then, $\beta>\left|\alpha_{1}\right|$, which is a contradiction. This implies that $A$ (resp. $B$ ) satisfies condition (i) in Theorem 3.1. Furthermore, by [13], $\operatorname{ext}\left(\mathcal{B}_{A_{d}^{*}}\right)=\left\{ \pm \delta_{x, x^{\prime}}: x, x^{\prime} \in X, x \neq x^{\prime}\right\}$ and, thus, $A$ and, similarly, $B$ satisfy the conditions in Theorem 3.1 .

Remark 3.4. There are many examples of extremely regular function spaces which are not algebras. For example, let $f$ be the function defined on $\mathbb{N}_{\infty}=\mathbb{N} \cup\{\infty\}$ as $f(n):=\frac{1}{n}$ for all $n \in \mathbb{N}$ and $f(\infty)=0$. Assume that $A$ is the $\mathbb{R}$-linear span generated by the set $\left\{1, f, \delta_{n}: n \in \mathbb{N}\right\}$. Then, clearly, $A$ is an extremely regular function space on $\mathbb{N}_{\infty}$ which is not an algebra. Indeed, if $A$ is an extremely regular function algebra on a compact space $X$ and $f$ is a function in $C(X)$ such that $f \neq f^{2}$ and $f, f^{2} \notin A$, then the $\mathbb{R}$-linear span generated by the set $\{f, A\}$ is an extremely regular function space which is not an algebra.

Let us recall that a simplicial function space is a point-separating closed linear (real-valued) subspace of $C(X)$ ( $X$ compact) which contains the constants and whose state space is a Choquet simplex.

Corollary 3.5. Let $A$ (resp. B) be a simplicial function space on a compact space $X$ (resp. $Y$ ). Assume that $T: A \rightarrow B$ is a surjective diameter preserving map. Then there are a homeomorphism $\psi: C h(B) \rightarrow C h(A)$, a scalar $\lambda \in\{ \pm 1\}$ and a linear functional $L: A \rightarrow \mathbb{R}$ such that for all $f \in A$ and $y \in C h(B), T f(y)=\lambda f(\psi(y))+L(f)$.

Proof. Let us first prove that $\left\{\delta_{x}: x \in X\right\}$ is linearly independent in $\left(A,\|\cdot\|_{\infty}\right)^{*}$. Suppose that there exist $\alpha_{1}, \cdots, \alpha_{n} \in \mathbb{R}$ and different points $x_{1}, \cdots, x_{n} \in X$ such that $\alpha_{1} \delta_{x_{1}}+\cdots+\alpha_{n} \delta_{x_{n}}=0$ on $A$. By [11, Theorem 19, I.23], $\left.A\right|_{\left\{x_{1}, \ldots, x_{n}\right\}}=C\left(\left\{x_{1}, \ldots, x_{n}\right\}\right)$, then for each $i, i=1, \cdots, n$, there is a function $f_{i} \in A$ with $f_{i}\left(x_{i}\right)=1$ and $f_{i}\left(x_{j}\right)=0$ for all $j \in\{1, \cdots, n\} \backslash\{i\}$. Thus we can conclude that $\alpha_{i}=0$. This means that $A$ (resp. $B$ ) satisfies condition (i) in Theorem 3.1.

On the other hand, since $A$ and $B$ are simplicial, we know, from [14] (see also [6]), that $\operatorname{dch}(A)=$ $\left\{\left\{x_{1}, x_{2}\right\}: x_{1}, x_{2} \in C h(A)\right\}$ and $d \operatorname{ch}(B)=\left\{\left\{y_{1}, y_{2}\right\}: y_{1}, y_{2} \in C h(B)\right\}$.

As a conclusion, by Theorem 3.1, we get the desired result. 


\section{REFERENCES}

[1] A. Aizpuru and F. Rambla, There's something about the diameter, J. Math. Anal. Appl. 330 (2007), 949-962.

[2] A. Aizpuru and M. Tamayo, Linear bijections which preserve the diameter of vector-valued maps, Linear Algebra Appl. 424 (2007), 371-377.

[3] A. Aizpuru and M. Tamayo, On diameter preserving linear maps, J. Korean Math. Soc. 45 (2008), 197-204.

[4] A. Aizpuru and F. Rambla, Diameter preserving linear bijections and $C_{0}(L)$ spaces, Bull. Belg. Math. Soc. Simon Stevin 17 (2010), 377-383.

[5] L. Asimow and A. J. Ellis, Convexity theory and its applications in functional analysis, Academic Press, London-New York, 1980.

[6] M. Bacak, Unique decomposition property and extreme points, Rocky Mountain J. Math. 39 (2009), 1397-1402.

[7] B. A. Barnes and A. K. Roy, Diameter preserving maps on various classes of function spaces, Studia Math. 153 (2002), 127-145.

[8] F. Cabello Sánchez, Diameter preserving linear maps and isometries, Arch. Math. 73 (1999), 373-379.

[9] F. Cabello Sánchez, Diameter preserving linear maps and isometries II, Proc. Indian Acad. Sci. (Math. Sci.) 110 (2000), 205-211.

[10] B. Cengiz, On extremely regular function spaces, Pacific J. Math. 49 (1973), 335-338.

[11] P. C. Curtis, Topics in Banach spaces of continuous functions, Lecture Notes Series n.25, 1970.

[12] J. J. Font and M. Hosseini, Diameter preserving mappings between function algebras, Taiwanese J. Math. 15 (2011), 1487-1495.

[13] J. J. Font and M. Sanchis, A characterization of locally compact spaces with homeomorphic one-point compactifications, Topology Appl. 121 (2002), 91-104.

[14] J. J. Font and M. Sanchis, Extreme points and the diameter norm, Rocky Mountain J. Math. 34 (2004), 1325-1331.

[15] F. González and V. V. Uspenskij, On homomorphisms of groups of integer-valued functions, Extracta Math. 14 (1999), 19-29.

[16] M. Győry and L. Molnár, Diameter preserving linear bijections of C(X), Arch. Math. 71 (1998), 301-310.

[17] G. M. Leibowitz, Lectures on complex function algebras, Scott, Foresman and Company, 1970.

[18] T. S. S. R. K. Rao and A. K. Roy, Diameter preserving linear bijections of function spaces, J. Aust. Math. Soc. 70 (2001), 323-335.

Department of Mathematics, K.N. Toosi University of Technology, Tehran, 16315-1618, Iran

E-mail address: m.hosseini@kntu.ac.ir

Departamento de Matemáticas, Universitat Jaume i, Campus Riu Sec, 8029 AP, Castellón, Spain

E-mail address: font@mat.uji.es 